\title{
A carte blanche approval in Alzheimer's
}

\author{
An Alzheimer's drug approval is being touted as a triumph for patients. But US healthcare is ill prepared for \\ Aduhelm's questionable efficacy, unfettered market access and high price.
}

A duhelm (aducanumab) is no cure for Alzheimer's disease. The efficacy of the drug in slowing cognitive decline of patients with prodromal or early-stage dementia is marginal at best. And high doses of the medicine are associated with an increase in brain swelling (at a rate of 35.2\% for the drug versus $2.7 \%$ for placebo). Yet on 7 June, contrary to the advice of its own scientific advisory committee, the US Food and Drug Administration (FDA) licensed the drug via the accelerated approval pathway (based on an ostensible surrogate marker of disease rather than proven clinical benefit).

The lax terms of Aduhelm's approvalincluding a nine-year timeline to demonstrate drug effectiveness and availability to patients with late-stage Alzheimer's-are baffling. On top of that, Biogen's annual price tag per patient of $\$ 56,000$ will not only wreak havoc on healthcare budgets but also increase health inequities, with many patients paying thousands of dollars out of pocket for a drug that may have little effect on their cognitive decline.

Aduhelm is one of a group of monoclonal antibodies, including donanemab,

lecanemab and gantenerumab, that target the $\beta$-amyloid fibrils implicated in Alzheimer's pathogenesis, but which until now have disappointed in the clinic. The antibody, which was out-licensed by Biogen from Neurimmune in 2007, is unique in being derived from human B cells from healthy elderly subjects with no signs of cognitive impairment and from cognitively impaired elderly subjects with unusually slow clinical decline.

Three trials (PRIME, ENGAGE and EMERGE) showed the drug lowers $\beta$-amyloid in a dose- and time-dependent manner. But Biogen and Eisai halted clinical testing after a futility analysis on interim data from ENGAGE and EMERGE suggested the trials were "unlikely to meet their primary [clinical] endpoint upon completion."

Several months later, there was an abrupt turnabout. Reanalysis of "additional data" from EMERGE showed that the drug had achieved a "significant $22 \%$ relative reduction in clinical decline"-a result later reportedly supported by "data from a subset of patients" in ENGAGE.

The FDA's Peripheral and Central Nervous System Drugs Advisory Committee begged to differ. A decisive vote against approval at its November 2020 meeting cited weaknesses in efficacy data and recommended that Biogen undertake a confirmatory trial.

Yet the FDA went ahead with approval, arguing to its committee members that there is "substantial evidence that the drug reduces $A \beta$ plaque, and that this reduction is reasonably likely to predict clinical benefit". Once the drug is prescribed in the general population, the presumption is that a stronger efficacy signal may emerge in a subgroup that was undetectable in clinical trials.

Of course, the FDA sometimes overrules scientific advice-between 2008 and 2015, it went against advisory committees $21 \%$ of the time. But for the most part it has been more (not less) conservative than its advisors.

No one doubts the need for new Alzheimer's drugs. The number of people affected is huge-6.2 million in the United States and 50 million worldwide-and existing treatments provide only temporary relief from cognitive symptoms. As illustrated by the 2016 approval of the Duchenne muscular dystrophy drug Exondys 51 (eteplirsen), the FDA is becoming increasingly receptive to pressure from patient advocacy groups. In the case of Aduhelm, the Alzheimer's Association and Us Against Alzheimer's pressed stridently for access.

From a patient perspective, the leniency of the FDA's requirements around the approval is understandable. But the approach is questionable in several ways, posing challenges to the US healthcare system that demand rapid action.

Aduhelm's broad label is the first problem. By failing to specify disease stage or require biomarker confirmation of amyloid positivity, the FDA has given Biogen and Eisai carte blanche to blitz the entire Alzheimer's market with a treatment of questionable efficacy for years or even decades. The agency could have restricted the use of the drug to early-stage disease (where the clinical data were gathered), knowing that physicians could still prescribe it off-label to other patients. But it didn't.

Neither is it clear that the drug is safe: brain swelling and bleeds are a risk with Aduhelm, and patients might be exposed for years without the guarantee of expensive serial MRI to monitor for them.

The third issue is that the FDA has given Biogen nine years to sell Aduhelm before completing a post-marketing trial that confirms it works-a period twice as long as the ENGAGE and EMERGE trials before approval. In any case, companies rarely complete post-marketing studies on time, often citing difficulties in recruiting treatment-naïve patients. They are rarely penalized for this; indeed, the FDA has never forced a drug withdrawal, let alone fined a company, for failing to complete a post-marketing commitment.

One potential solution might be for the US CMS Innovation Center to launch its own study of Aduhelm outcomes. Players like AARP and United HealthCare might also negotiate value-based contracts with Biogen whereby full reimbursement is triggered only when claims data show the therapy works. But this is unlikely to be feasible in a million-patient market.

The FDA's 7 June decision means that payers and patients now must deal with a drug of questionable efficacy priced at nearly ten times the level recommended by Institute for Clinical and Economic Review. The drug will bring substantial extra costs if it requires diagnostic PET procedures versus cerebrospinal fluid-based or blood-based testing. Payers will likely not offer full coverage for Aduhelm, meaning patients and their families must fund the difference.

If Aduhelm is a signpost of the way regulation is moving-accelerated approvals using surrogate markers and limited clinical efficacy data in chronic disease-then US reimbursement must adapt and become outcome based. Regulation, market access and payment are all intertwined. A solution must be found that balances increased market access with patient protections, outcomes and costs.

Published online: 28 June 2021

https://doi.org/10.1038/s41587-021-00995-4 